\title{
Fluorescence imaging contrast in guided surgery on nerves measured in rats in vivo
}

Fanjul-Vélez, F., Díaz-Martínez, A., Garro-Martínez, E., Arce-Diego, J.

F. Fanjul-Vélez, A. M. Díaz-Martínez, E. Garro-Martínez, J. L. Arce-Diego, "Fluorescence imaging contrast in guided surgery on nerves measured in rats in vivo," Proc. SPIE 11222, Molecular-Guided Surgery: Molecules, Devices, and Applications VI, 112220K (19 February 2020); doi: 10.1117/12.2544665 


\title{
Fluorescence imaging contrast in guided surgery on nerves measured in rats in vivo
}

\author{
F. Fanjul-Vélez*a, A. M. Díaz-Martínez ${ }^{\mathrm{b}, \mathrm{c}, \mathrm{d}}$, E. Garro-Martínez ${ }^{\mathrm{a}, \mathrm{b}}$, J. L. Arce-Diego ${ }^{\mathrm{a}}$ \\ aApplied Optical Techniques Group, TEISA Department, University of Cantabria, Av de los Castros \\ s/n, 39005 Santander, Spain \\ ${ }^{\mathrm{b}}$ Centro de Investigación Biomédica en Red de Salud Mental (CIBERSAM), Carlos III Health \\ Institute, Santander, Spain \\ ${ }^{c}$ Cantabria Biomedicine and Biotechnology Institute (IBBTEC), University of Cantabria-CSIC- \\ SODERCAN, Avda. Albert Einstein, 22, 39011, Santander, Spain \\ ${ }^{\mathrm{d} P h y s i o l o g y}$ and Pharmacology Department, Faculty of Medicine, University of Cantabria, \\ Santander, Spain
}

\begin{abstract}
An appropriate identification of the tissue type that is in front of the excision surgical tool is critical, for either tumoral tissue removal, or even accessory healthy tissues whose destruction could cause severe collateral damage. Healthy tissue distinction is an unsolved task in real surgical praxis, due for instance to alterations or hemorrhages. Many approaches have tried to solve the problem of healthy tissue discrimination, such as spectroscopy. Diffuse Reflectance Spectroscopy (DRS), an easy to implement and reliable optical diagnostic technique, has shown great potential in tumoral tissue discrimination, as long as in healthy tissue distinction. However, the identification of nerve tissue in particular is challenging. What is more, nerve tissue distinction is critical in surgical interventions, due to the severe irreversible dysfunctions in patient mobility that collateral damage could cause. Fluorescence is an optical technique that could be of relevance for nerve identification. The need of specific fluorophores that tend to accumulate preferentially on nerve tissue is essential for this task. Image quality requires also an analysis of the optical source, filters employed, fluorophore concentration or inoculation method. Together with those aspects, additional open questions include incubation time or cytotoxicity, critical for clinical translation. In this work, fluorescence imaging for nerve identification is applied on in vivo rats. The approach uses several fluorophore concentrations, based on Oxazine, and combines topical and intravascular inoculation. Optical irradiance is considered for image contrast. The results show an appreciable nerve contrast for optimized parameters.
\end{abstract}

Keywords: fluorescence, biomarkers, nerve tissue, in vivo animal imaging, guided-surgery

\section{INTRODUCTION}

The applications of optics in medical practice are based on the study of biological light-tissue interaction. Most of the biological tissues can be defined, from the optical point of view, as multilayered turbid media. This definition implies the presence of absorption properties and high scattering. Different effects can be distinguished in the interaction between optical radiation and biological tissues. Among the most relevant ones in optical techniques applied to biomedical practice are reflection and transmission, as well as the aforementioned scattering and absorption. The propagation of optical radiation in a biological tissue can be estimated from the above effects, among others. The relevance of these effects must be first quantified, in order to carry out analysis and designs. Typically, the so-called optical properties, such as refractive index, absorption coefficient, scattering coefficient, or scattering anisotropy, are used to characterize biological tissues [1]. The property of absorption is particularly relevant, as it greatly influences the penetration depth of optical radiation. For example, it appears as fundamental in the design of a certain diagnostic or treatment equipment. It is mainly absorption that defines the so-called therapeutic window. This range of wavelengths, between 600 and $1100 \mathrm{~nm}$, represents the spectral region with the lowest attenuation in biological tissues.

*fanjulf@unican.es; arcedj@unican.es phone +34 9422067 30; www.teisa.unican.es/toa

Molecular-Guided Surgery: Molecules, Devices, and Applications VI, edited by Sylvain Gioux,

Summer L. Gibbs, Brian W. Pogue, Proc. of SPIE Vol. 11222, 112220K · @ 2020 SPIE

CCC code: $1605-7422 / 20 / \$ 21 \cdot$ doi: $10.1117 / 12.2544665$

Proc. of SPIE Vol. 11222 112220K-1 
One of the most critical diagnostic problems in clinical practice has to do with conventional biopsy, a gold standard diagnostic approach. Optical techniques could be an alternative of interest in order to try to avoid its invasive nature, as well as diagnostic delays. In this way, there would be non-invasive, non-contact, non-ionizing and high resolution and contrast techniques, for instance for pathological tissue identification [2]. Success in this goal would lead to the so-called optical biopsy, which could revolutionize clinical practice. In the particular case of diagnosis, there is a currently unsolved application that could also benefit from the advantages of optical techniques. In particular, the application refers to guided surgery systems, which require tissue discrimination before addressing the corresponding procedures, usually selective cutting. Optical surgery provides a localized and controlled tissue excision [3]. In addition to achieving an accurate intervention, tissue type discrimination would prevent damage to adjacent tissues. The damage can be particularly relevant if these adjacent tissues are, for example, blood vessels or nerve tissue.

Among the optical techniques that could contribute to solving this problem are spectroscopic techniques. As mentioned earlier, the optical radiation that absorbs and scatters a sample depends strongly on its optical properties, mainly on its absorption coefficient $\mu_{a}$ and reduced scattering $\mu_{s}{ }^{\prime}$. Consequently, the spectral information of a tissue contains diagnostic data. Numerous studies have demonstrated the feasibility of the technique to determine the state of a tissue and provide information on the morphology, functionality or biochemical composition of the sample $[4,5]$. The intrinsic contrast achieved with techniques such as Diffuse Reflectance Spectroscopy is not always sufficient [6], and therefore it is necessary to include some extrinsic substance to try to increase it. Among the most common exogenous components are fluorophores, widely used in biological systems, as well as in the clinics. Specific substances could then serve as distinctive elements in the mentioned applications of guided surgery. This substances could even be used for treatment and monitorization $[7,8]$. The identification of nerve tissue is particularly critical to this aim, as unintended sectioning could provoke severe damage to the patient. Among the techniques intended for nerve identification, fluorescence is probably the most promising one [9]. Specific fluorophores tend to accumulate preferentially on nerve tissue, and contribute, as a consequence, to tissue discrimination. Although preliminar results are promising, there are still open questions regarding signal to background acceptable ratios, incubation time or cytotoxicity that need to be solved prior to clinical translation.

In this work, fluorescence imaging is applied to nerve identification in in vivo rats. The application is made by topical and intravascular approaches. Several fluorophore concentrations are employed, and the influence of the solvent is analyzed. Section 2 presents the main mechanisms of fluorescence spectroscopy. In section 3 some results are presented and discussed. The fourth section contains the conclusions.

\section{FLUORESCENCE SPECTROSCOPY}

Fluorescence is used in biomedical applications mainly for the diagnosis of biological tissues [7, 8]. There are certain intrinsic substances in biological tissues capable of producing the effect of fluorescence. In this case the effect is known as self-fluorescence. In case the contrast is not enough, or there are no fluorescent substances, extrinsic fluorophores are used. In medical practice the second approach is more common. Two extrinsic fluorophores approved for clinical use are Methylene Blue and Indocyanine Green. Both have the approval of the American FDA (Food and Drug Administration), and are commonly used in clinical practice, especially for the detection of tumor tissues. The spectral response is essential in the characterization of fluorophores, since this response is subsequently used as a differentiating element in the diagnosis. In addition, the spectral response determines the quantum efficiency, and therefore the irradiance of the emitted radiation, which constitutes a key aspect of the design of equipment for fluorescence measurements. In this way it is possible to facilitate the location of specific points of the anatomy that would otherwise be much more difficult, or even impossible, to locate.

The fluorophores under analysis are pure solids, which makes it necessary to use solvents for clinical use. The clinical administration is usually systemic, intravenous, or sometimes topical. In any case, the administration medium is usually a liquid or an aerosol. Fluorophore concentration and solvent type generate different interactions, which give rise to significant variations in the excitation spectrum. These variations greatly modify the characteristics of the absorbed and emitted radiation, which makes both the efficiency and the spectral configuration of the diagnostic detection system completely change.

The absorption of light, which is mainly responsible for fluorescence, occurs mainly through three basic processes: electronic transitions (between atoms and molecules, typically between ultraviolet and infrared), vibrational transitions (only between molecules, infrared region) and rotational transitions (only between molecules, infrared and submillimeter 
wavelengths). The efficiency of light absorption at a wavelength $(\lambda)$ by an absorbent medium is characterized by the absorbance $\mathrm{A}(\lambda)$ or by the transmittance $\mathrm{T}(\lambda)$ defined in equations 1 and 2 respectively [1].

$$
\begin{gathered}
(\lambda)=\log \left(\frac{I_{\lambda}^{0}}{I_{\lambda}}\right)=-\log T(\lambda) \\
T(\lambda)=\frac{I_{\lambda}^{0}}{I_{\lambda}}
\end{gathered}
$$

Where $I_{\lambda}^{0}$ and $I_{\lambda}$ represent the average light intensity of the incident and transmitted beams, respectively. In many cases, absorbance follows the Beer-Lambert law:

$$
A(\lambda)=\varepsilon(\lambda) \cdot l \cdot c
$$

Where $\varepsilon(\lambda)$ is the molar absorption coefficient expressed in $\mathrm{L} /(\mathrm{mol} \cdot \mathrm{cm})$, c is the concentration of the species absorbed in $\mathrm{mol} / \mathrm{L}$, and $\mathrm{l}$ is the length of the optical path expressed in $\mathrm{cm}$. In order to check if fluorescent discrimination of nerve tissue is possible, it is necessary to carry out experiments in vivo. In this way it is possible to perform a first evaluation of the feasibility of the technique.

\section{RESULTS AND DISCUSSION}

The fluorophore employed must present preferential accumulation in nerve tissue, as some oxazines do. The imaging setup is based on a previous knowledge of the spectroscopic characteristics of the fluorophore, and are a function of the solute employed or fluorophore concentration. This last parameter alters the total intensity signal, which is directly proportional to the amount of molecules, and also spectral absorption and emission, as chemical bonding changes with an increasing number of molecules. In this work two different solutes were employed. One of them was appropriate for the topical application, and the other one for intravascular inoculation. Figure 1 shows an example of emission spectra of Oxazine 4 at a concentration of $20 \mu \mathrm{M}$ in water and in Phosphate Buffered Saline (PBS).

Measurements were made on in vivo Sprague Dawley rats. The experimental protocol was approved by the bioethical committee of the University of Cantabria. Rats remained at least a week at the University facilities until they reach a weight of around 150-200 grams. They were anesthetized by pentobarbitone at $65 \mathrm{mg} / \mathrm{kg}$. Fluorophore preparations included topical and intravascular administration. Bilateral sciatic nerves were exposed by open surgery at the predetermined incubation times. The imaging setup included a high power LED at $630 \mathrm{~nm}$, excitation and emission filters, a dichroic, a CMOS camera and a camera objective.

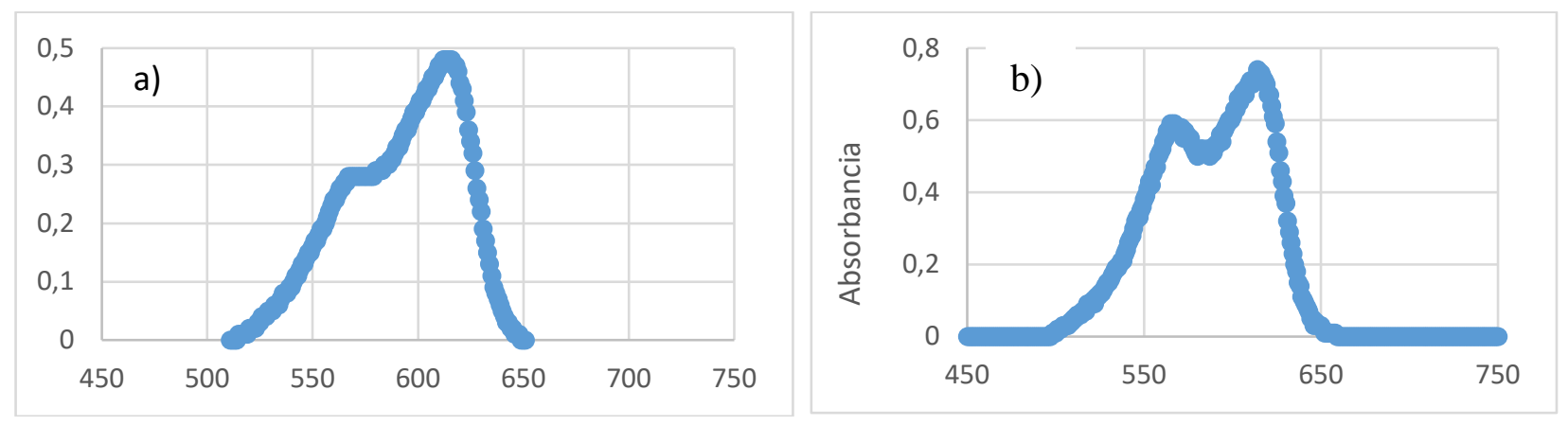

Figure 1. Emission spectra of Oxazine 4 at $20 \mu \mathrm{M}$ in a) water and b) PBS.

Fluorescence imaging of the exposed sciatic nerve is shown in Figure 2. White light image appears in Figure 2a, and fluorescence image in Figure 2b. The sciatic nerve is clearly identified in the fluorescence image over the background, although exposed areas that correspond to cut muscle tissue also present significant signal. The rest of fat and muscle tissue remain with low fluorophore concentration. Several concentrations, irradiances and incubation times were measured. The results contribute to nerve discrimination in guided surgery. 


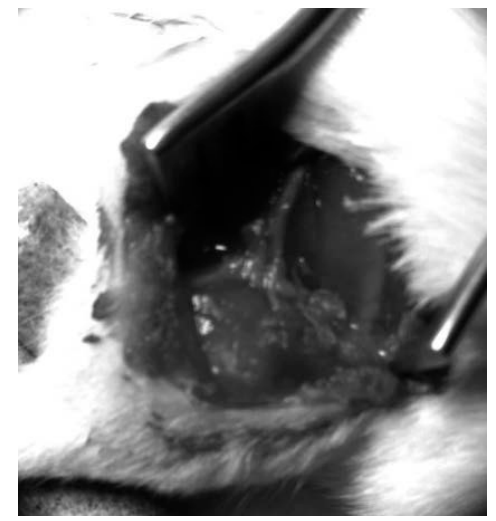

a)

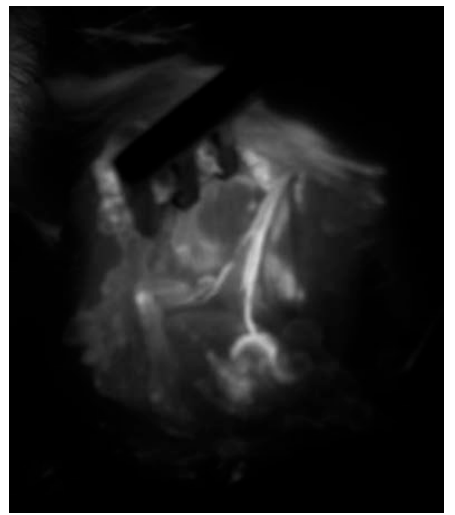

b)

Figure 2. Example of sciatic nerve imaging in a) white light image and b) fluorescence image.

\section{CONCLUSIONS}

In this work tissue discrimination for guided surgery has been explored. In particular, the problem of identifying nerve tissue has been analyzed and tested. Endogenous Diffuse Reflectance Spectroscopy has been shown as an appropriate technique for tissue discrimination, but the identification of nerve tissue remains an issue. Exogenous contrast for nerve tissue has been tested on in vivo rats by means of specific fluorophores. The influence of fluorophore concentration and solvents has been studied and experimentally tested. Fluorescence imaging was applied to validate the results. The proposed technique could contribute to the reduction of collateral damage on nerve tissue in guided surgery, avoiding severe consequences of accidental nerve resection.

\section{ACKNOWLEDGEMENTS}

This work has been partially supported by the project "High-pressure driven plasmonic and luminescence properties of naked and core/shell metal-oxide nanocomposites” (PGC2018-101464-B-I00) of the Spanish Ministry of Science, Research and Universities, cofunded by FEDER funds, and by the San Cándido Foundation.

\section{REFERENCES}

[1] Niemz, M. H., [Laser-tissue interactions: fundamentals and applications], Springer Science \& Business Media, Heidelberg, (2013).

[2] Fanjul-Vélez, F., Pircher, M., Baumann, B., Götzinger, E., Hitzenberger, C. K. and Arce-Diego, J. L., "Polarimetric analysis of the human cornea by Polarization Sensitive Optical Coherence Tomography," J. Biomed. Opt. 15, 056004 (2010).

[3] Fanjul-Vélez, F., Salas-García, I. and Arce-Diego, J. L., "Analysis of laser surgery in non-melanoma skin cancer for optimal tissue removal”, Laser Physics 25, 025606 (2015).

[4] Koenig, F., Larne, R., Enquist, H., McGovern, F. J., Schomacker, K. T., Kollias, N. and Deutsch, T. F., "Spectroscopic measurement of diffuse reflectance for enhanced detection of bladder carcinoma," Urology 51(2), 342-345 (1998).

[5] Zonios, G., Perelman, L. T., Backman, V., Manoharan, R., Fitzmaurice, M., Van Dam, J. and Feld, M. S., "Diffuse reflectance spectroscopy of human adenomatous colon polyps in vivo,” Applied Optics 38(31), 6628-6637 (1999). 
[6] Fanjul-Vélez, F., Arévalo-Díaz, L. and Arce-Diego, J. L., "Intra-class variability in diffuse reflectance spectroscopy: application to porcine adipose tissue,” Biomedical Optics Express 9, 2297-2303 (2018).

[7] Salas-García, I., Fanjul-Vélez, F. and Arce-Diego, J. L., "Spatial photosensitizer fluorescence emission predictive analysis for photodynamic therapy monitoring applied to a skin disease," Optics Communications 285, 15811588 (2012).

[8] Salas-García, I., Fanjul-Vélez, F. and Arce-Diego, J. L., "Superficial radially-resolved fluorescence and threedimensional photochemical time-dependent model for Photodynamic Therapy,” Optics Letters 39, 1845-1848 (2014).

[9] Shaefer, J. M., Barth, C. W., Davis, S. C. and Gibbs, S. L., "Diagnostic performance of receptor-specific surgical specimen staining correlates with receptor expression level," Journal of Biomedical Optics 24, 026002 (2019). 